\title{
Clinicopathological overview of thrombocytopenia: A retrospective study in tertiary care hospital
}

\author{
Mukesh Baheti ${ }^{1}$, Dhiraj Nikumbh ${ }^{2, *}$, Sushma Desai $^{3}$ \\ ${ }^{\mathbf{1}}$ Assistant Professor, ${ }^{2}$ Professor, ${ }^{3}$ Ex. Professor, ${ }^{\mathbf{1}}{ }^{3}$ Dept. of Pathology, ${ }^{1}$ Prakash Institute of Medical Sciences, Islampur, \\ Maharashtra, ${ }^{2}$ ACPM Medical College, Dhule, ${ }^{3}$ Krishna Institute of Medical Sciences, Karad, Maharashtra, India
}

*Corresponding Author:

Email: drdhirajnikumbh@ rediffmail.com

\begin{abstract}
Introduction: Thrombocytopenia is not a disease itself, but a subnormal number of platelets in blood and is one of the most common causes of abnormal bleeding. The patient with thrombocytopenia often presents diagnostic and management challenges. The differential diagnosis is broad because the disorders leading to thrombocytopenia are diverse, with failed production at one extreme and the accelerated destruction at the other.

Aims and Objective: To highlight and diagnose thrombocytopenia and investigate the cause of thrombocytopenia in our tertiary care center.

Materials and Methods: The present study is retrospective, cross sectional study done on screened 389 thrombocytopenia patients which were came to our hematology lab. All patients of proved thrombocytopenia patients were included in the study over a period of 3 years from May 2013 to April 2016. Pseudo-thrombocytopenia patients were excluded from the study. All these patients were investigated in a systemic manner, causes of thrombocytopenia were ascertained and data was analyzed.

Results: The most common affected age group of thrombocytopenia was $3^{\text {rd }}$ decades and $2^{\text {nd }}$ decades respectively with male predominance. The most common cause of thrombocytopenia is anemia, infection, spleenomegaly and alcoholism. Dimorphic anemia, malaria and HIV are the most etiology of anemia and infection respectively.

Conclusion: Thrombocytopenia is root cause of abnormal bleeding in many cases. Importance of early and prompt diagnosis of thrombocytopenia is always warranted as treatment differs depending upon the etiology. Thrombocytopenia is multifactorial and a finding that may result from various diseases and clinicopathological approach is important in these cases.
\end{abstract}

Keywords: Thrombocytopenia, Platelets, Etiology, Multifactorial, Clinicopathological.

\section{Introduction}

Thrombocytopenia is not a disease entity by itself, but a finding that may result from a number of disease processes. Thrombocytopenia may be defined as subnormal number of platelets in the circulating blood and is one of the most common causes of abnormal bleeding. ${ }^{1}$ The patient with thrombocytopenia often presents diagnostic and management challenges simultaneously. The differential diagnosis is broad because the disorders leading to thrombocytopenia are diverse, with failed production at one extreme and the accelerated destruction at the other. ${ }^{2}$

Presentation of thrombocytopenic patients varies, most being devoid of symptoms and others may present with minor to severe fatal bleeding. ${ }^{3}$ Though there is no absolute relationship, degree of thrombocytopenia correlates with risk of bleeding. The condition under which it has developed has an important influence on the occurrence of bleeding. ${ }^{4}$ Besides history and proper examination, a number of tests are available to arrive at the etiology of thrombocytopenia. However one must use clinical judgment to choose between them. ${ }^{3}$ The management of thrombocytopenia differs widely depending on the cause, hence the importance of early and accurate diagnosis.

We were prompted to undertake this study with the aim of diagnosis the cases of thrombocytopenia with their causes because despite the research into platelet structure and function, the commonest platelet abnormality encountered in clinical practice is quantitative reduction of them i.e. thrombocytopenia and it is this condition, which causes the greatest morbidity and mortality in platelet-associated disease.

\section{Materials and Methods}

The present study is retrospective, cross sectional study done on screened 389 thrombocytopenia patients which were came to our hematology lab. All patients of proved thrombocytopenia patients were included in the study over a period of 3 years from May 2013 to April 2016. Pseudo-thrombocytopenia patients were excluded from the study. All these patients were investigated in a systemic manner, causes of thrombocytopenia were ascertained and data was analyzed.

All the patients referred to the central clinical laboratory for routine complete blood count and peripheral blood smear examination were screened for thrombocytopenia and total number of 389 cases were selected based on standard criteria as- Platelet count below 1.5 lakh/cmm. ${ }^{4}$ Complete medical history was taken and detailed physical examination was done for each patient with special attention to the signs of bleeding from any site. Bone marrow examination was done whenever necessary and indicated. Many other investigations like- reticulocyte count, erythrocyte sedimentation rate (ESR), urine and stool examination, 
liver function tests, USG abdomen, lupus erythematous cell test, urinary Bence Jones proteins, serum protein electrophoresis, bone and chest radiographs etc. were done whenever indicated.

Hemoglobin level, leucocyte and platelet counts were done by electronic cell counter SYSMEX KX-21 that works on aperture impendence technology. In all cases CBC bulb was checked again for absence of blood clot and clumps on PBS to rule out pseudothrombocytopenia. Hemoglobin level, leucocyte and platelet count done by the cell counter were reassessed during peripheral smear examination by Leishman stain. Platelet count was also done on Improved Neubaur chamber by Rees and Ecker method. Stained peripheral smear is screened for presence of platelet aggregates or platelet satteletism, in the presence of which it is not possible to estimate the exact number of platelets. In an area on smear where red cells are just beginning to overlap each other, using a 1000X magnification, each platelet seen is equivalent to 15000 on the total platelet count. Mean number of platelets in 10 such representative fields is calculated and total platelet count is calculated.

All the patients thus selected were investigated in a systemic manner, causes of thrombocytopenia were ascertained and data was analyzed.

\section{Result and Observations}

Though thrombocytopenia is not a disease by itself, it is a striking feature of many disorders, many of which are very common and easily treatable like megaloblastic anemia, infections like malaria, and others may be very serious and life threatening like various leukemias, multiple myeloma etc.

There are very few comprehensive studies on this subject, though extensive studies have been done for the different etiological factors like ITP, aplastic anemia, HIV, other infections etc.

The age range in our study was 1 day to 95 years. The maximum numbers of cases i.e. 149(38.30\%) were in $3^{\text {ed }}$ and $4^{\text {th }}$ decade. A definite male preponderance was seen in the overall picture as well as in almost all age groups. Male outnumbered females because prevalence of major etiological factors in our study like megaloblastic anemia, infections like HIV and malaria, congestive spleenomegaly and alcoholism is more in males than in females. Lakhotia $\mathrm{M}$ et $\mathrm{al}^{5}$ had shown that the prevalence of megaloblastic anemia is more in males.

In many instances more than one pathogenetic factor may be responsible for thrombocytopenia. ${ }^{6-8}$ Our findings are in keeping with the above statement. We found $150(38.56 \%)$ cases of thrombocytopenia, where multiple factors were contributing. This could be because of high prevalence of major etiological factors like anemia, infection, and alcoholism in rural population of India.

Stephan $\mathrm{F}$ et $\mathrm{al}^{9}$ found combination of multiple factors contributing to thrombocytopenia in one quarter of patients in surgical ICU. In our study commonest cause contributing to thrombocytopenia was anemia in $166(42.67 \%)$ cases followed by infection, hypersplenism and alcoholism in 152(39.07\%), $70(17.99 \%)$ and $67(17.22 \%)$ cases respectively. Hematological malignancies in 33(8.48\%) cases, use of myelosuppressants in $28(7.16 \%)$ cases, snakebite in 23(5.19\%), hypertensive disorders of pregnancy in $17(4.37 \%)$ cases, DIC in $12(3.08 \%)$ cases and ITP in $9(2.31 \%)$ cases were the other factors contributing to thrombocytopenia.

We came across only two such comprehensive studies on thrombocytopenia as Table 1 .

Table 1: Comparative study of major causes

\begin{tabular}{|l|c|c|c|}
\hline & $\begin{array}{c}\text { Ross C et a } \mathbf{~}^{\mathbf{1 0}} \\
\mathbf{1 1 0} \text { cases }\end{array}$ & $\begin{array}{c}\text { Alam M et } \text { al }^{\mathbf{1 1}} \\
\mathbf{5 0 0} \text { cases }\end{array}$ & $\begin{array}{c}\text { Our study } \\
\mathbf{3 8 9} \text { cases }\end{array}$ \\
\hline $1^{\text {st }}$ common cause & Anemia $38.2 \%$ & Malaria $43.2 \%$ & Anemia $42.6 \%$ \\
\hline $2^{\text {nd }}$ common cause & ITP $10.9 \%$ & Viral infections & Infection $39.07 \%$ \\
\hline
\end{tabular}

Ross c et $\mathrm{al}^{10}$ had found anemia as a major contributing factor for thrombocytopenia. Similar is the case with our study, anemia contributing in $166(42.67 \%)$ cases. Alam et al. ${ }^{11}$ had found malaria and other infections as a major cause for thrombocytopenia. Infection ranked second only next to anemia in the list of causes of thrombocytopenia in our study. Infection is the most common cause of thrombocytopenia in newborns while acute leukemia and infection are the major causes of thrombocytopenia in childhood. ${ }^{12}$ Infection is known to produce thrombocytopenia either by increased destruction or decreased production of platelets. Guida JD et al. ${ }^{13}$ had demonstrated thrombocytopenia in 54\% of neonates with sepsis.
In one analysis alcohol was contributing factor in $26 \%$ of patients admitted to hospital with platelet count of $<100,000 / \mathrm{cmm}^{14}$ In our study alcohol had contributed to $17.22 \%$ of thrombocytopenic patients. Although alcohol abuse results in thrombocytopenia in majority of cases by ineffective production related to folate deficiency and increased splenic pooling due to portal hypertension, it may occur in patients with alcoholism without accompanying vitamin deficiency, anemia or spleenomegaly. ${ }^{14,15}$ Transient thrombocytopenia related to heavy drinking bouts may also occur. ${ }^{4}$

Finding in our study were keeping with the above statement. We found that majority of the cases that is $11(84.62 \%)$ out of 13 of thrombocytopenia in neonates 
were due to sepsis. Infection and acute leukemia (ALL) were the major causes of thrombocytopenia in $5(38.46 \%)$ and $4(30.77 \%)$ cases respectively in children of 1 to 10 years age group. Rest of the findings are summarized in Table 2.

Table 2: Classification of various causes of Thrombocytopenia

\begin{tabular}{|c|l|c|c|}
\hline S. No & \multicolumn{1}{|c|}{ Causes } & $\begin{array}{c}\text { Number of cases as } \\
\text { isolated etio/associated } \\
\text { with other= Total }\end{array}$ & $\begin{array}{c}\text { Percentage } \\
(\mathbf{\%})\end{array}$ \\
\hline 1 & Anemia & $55 / 111=166$ & 42.67 \\
\hline 2 & Infection & $57 / 95=152$ & 39.07 \\
\hline 3 & Hypersplenism & $6 / 64=70$ & 17.99 \\
\hline 4 & Alcoholism & $15 / 52=67$ & 17.22 \\
\hline 5 & Hemat malignancies & $31 / 2=33$ & 8.45 \\
\hline 6 & $\begin{array}{l}\text { Use of } \\
\text { myelosuppressants }\end{array}$ & $27 / 1=28$ & 7.19 \\
\hline 7 & Snake bite & $22 / 1=23$ & 5.91 \\
\hline 8 & $\begin{array}{l}\text { Pregnancy induced } \\
\text { Hypertension }\end{array}$ & $12 / 5=17$ & 4.37 \\
\hline 9 & DIC & $1 / 11=12$ & 3.08 \\
\hline 10 & ITP & $8 / 1=9$ & 2.31 \\
\hline 11 & Miscellaneous & $5 / 2=7$ & 1.80 \\
\hline
\end{tabular}

\section{Discussion}

Platelets (literally, a small plate), also known as thrombocyte (thrombos, lump or clot; kytos, cell) circulate as cytoplasmic discs. ${ }^{6,16}$ On Wright-Giemsa stained blood smears platelets appear as small anucleate rounded or ellipsoidal discs. ${ }^{1,17}$ The cytoplasm is clear blue [hyalomere (hyalo-glass, meros-part)] and contains fine azure granules [chromatomere or granulomere]. ${ }^{7,18}$ Average volume of platelets is $7.06 \pm 4.85 \mathrm{f} 1$, with Diameter-3.6 $\pm 0.7 \mathrm{um}$ And Thickness $-0.9 \pm 0.3 \mathrm{um} ., 18$

The platelet membrane is a typical trilammenar membrane that is covered with an amorphous coat, 10$20 \mathrm{~nm}$ thick. The composition of this contact reactive coat includes glycoproteins, sulph-hydryl containing proteins, adenyl cyclase, glycosyl transferases, actomyosin \& adsorbed plasma proteins (especially the coagulation factors fibrinogen, and factors V, VII, XI). The plasma membrane forms a sponge like network of tortuous surface connecting channels that expands the surface area of platelet membrane enormously and serves as conduct for the extrusion of secretory products. The dense tubular system, derived from endoplasmic reticulum, interdigitates with the surface connecting system. ${ }^{6}$

Platelet cytoskeleton contain 30 to $50 \%$ of total platelet protein and is made up of three major structural components: an actin microfilament network present throughout the cytoplasm, a microtubule coil localized at the periphery, and a membrane skeleton comprising a network of short actin filaments that underlay the inner surface of plasma membrane. ${ }^{1}$

Platelets contain four distinct populations of granules: $\alpha$-granules, dense bodies, lysosomes and microperoxisomes. ${ }^{19}$ Thrombopoiesis- Two alternative processes have been proposed.
The first proposal is that platelets are preformed within the megakaryocyte by the demarcation membrane system; a series of membranes extending throughout the cytoplasm over the mature megakaryocyte. ${ }^{19}$ The second theory proposes that megakaryocyte cytoplasm undergoes sequential binary division. The naked megakaryocyte nuclei undergo phagocytosis by reticulo-endothelial cells. Thus megakaryocytes are suicidal micro-organs whose mission is to proliferate and fragment their cytoplasm on demand to maintain blood platelets at relatively steady levels. ' 'Thrombocytopenia'

Thrombocytopenia is defined as a reduction in the peripheral platelet count below the lower normal limit of $150 \times 10^{9} / \mathrm{L} .^{4}$ It is the most common cause of abnormal bleeding. The pathophysiology of thrombocytopenia is similar to that of anemia, but the later is better understood. Thus, despite the number and diversity of disorders that may be associated etiologically, thrombocytopenia results from only four processes: artifactual thrombocytopenia, deficient platelet production, accelerated platelet destruction and abnormal platelet distribution or pooling of the platelets within the body. ${ }^{1}$ It must be recognized that in many instances more than one pathogenetic factor may be responsible for thrombocytopenia, and that in many other cases the exact cause is unknown. ${ }^{7}$

Although it is difficult to judge the exact risk of bleeding, the following general guidelines seen to be adequate. ${ }^{3,20}$

1. Platelet count above $100,000 / \mathrm{cmm}$ is associated with normal haemostasis.

2. Platelet count between $50,000 / \mathrm{cmm}$ and $100,000 / \mathrm{cmm}$ should have no increased risk of spontaneous or surgical bleeding. 
3. Platelet count between 20,000/cmm and $50,000 / \mathrm{cmm}$ has an increased risk of surgical bleeding but a minimal risk of spontaneous bleeding.

4. Platelet count below $20,000 / \mathrm{cmm}$ depending on the cause, are often associated with an increased risk of spontaneous bleeding and more severe hemorrhage following surgical procedures and injuries.

\section{Pathophysiological thrombocytopenia ${ }^{1}$}

\section{classification}

of

1. Artifactual thrombocytopenia

a. Platelet clumping caused by anticoagulantdependant immunoglobulin. (Pseudo thrombocytopenia)

b. Platelet satellitism.

c. Giant platelets.

2. Decreased platelet production.

a. Hypoplasia of megakaryocytes.

b. Ineffective thrombopoiesis.

c. Disorders of thrombopoietic control.

d. Hereditary thrombocytopenias.

3. Increased platelet destruction.

a. Caused by immunological processes.

i. Autoimmune

ii. Idiopathic

4. Secondary- infections, pregnancy, collagen vascular disorders, drugs, miscellaneous.

a. Alloimmune-

i. Neonatal thrombocytopenia.

ii. Post-transfusion purpura.

b. Caused by nonimmunological processes.

i. Thrombotic microangiopathies.

a) Disseminated intravascular coagulation.

b) Thrombotic thrombocytopenic purpura.

c) Hemolytic-uremic syndrome.

c. Platelet damage by abnormal vascular surfaces Miscellaneous.

i. Infection.

ii. Massive blood transfusions.

5. Abnormal platelet distribution or pooling

a. Disorders of spleen.

b. Hypothermia.

c. Dilution of platelets with massive transfusions.

If all elements of complete blood count are considered in clinical context including findings of physical examination, they can provide valuable information guiding to probable cause of thrombocytopenia and helping in planning the workup of tests needed for definite diagnosis. Unnecessary test not only add to the expense of treatment but also may result in delayed diagnosis and inappropriate treatment in some cases.

As physicians who are not hematologists are not likely to be as well grounded in the specific constellation of finding that characterize individual hematological entities; stringency of diagnostic criteria and a general conceptual framework for ascertaining the cause of thrombocytopenia is of value and a demand of time.

\section{Summarized main findings}

1. The commonest age groups for thrombocytopenia were between $31-40$ and 21-30 years.

2. A definite male preponderance was seen in overall picture as well as in almost all age groups. The overall male to female ratio being 2.04:1.

3. Anemia followed by infection, hypersplenism and alcoholism were the common causes of thrombocytopenia in our study.

4. Out of 389 cases, in $150(38.56 \%)$ cases multiple factors contributing to thrombocytopenia were found.

5. In infants, infection was major cause of thrombocytopenia.

6. In $1^{\text {st }}$ decade of life, infection and hematological malignancy (ALL) were the major causes of thrombocytopenia.

7. The maximum number of cases of anemia and infection were in $3^{\text {ed }}$ and $4^{\text {th }}$ decade of life.

8. The maximum numbers of cases of myelosuppression by chemo/radiotherapy were found in $5^{\text {th }}$ and $6^{\text {th }}$ decade of life.

9. Dimorphic anemia and megaloblastic anemia were the major contributing factors for thrombocytopenia in anemic patients.

10. Though congestive splenomegaly and malarial infection were the common causes of hypersplenism other causes like tropical splenomegaly, typhoid fever and other infections, infiltration by NHL were also found.

11. Malaria, HIV, septicemia, other bacterial and viral infections can cause thrombocytopenia.

12. Bone marrow examination has definite role in ascertaining the various causes of thrombocytopenia like ITP, dimorphic and megaloblastic anemia, aplastic / hypoplastic anemia, various leukemia, plasma cell dyscrasia, MDS and lymphoma infiltrating marrow.

13. In majority of cases of thrombocytopenia due to isolated anemia, infection, splenomegaly, alcoholism and hypertensive disorders of pregnancy platelet count was $>50000 / \mathrm{cmm}$.

14. In majority of cases of thrombocytopenia due to hematological malignancy platelet count was $<50000 / \mathrm{cmm}$ with increased risk of bleeding.

15. There was bleeding in $9.77 \%$ of thrombocytopenic patients and we found that the risk of bleeding increases as platelet count decreases.

16. Major site of bleeding was G.I.T. and some of the patients had bleeding from multiple sites.

17. In many cases of bleeding besides thrombocytopenia other associated factors like functional defects of platelets, coagulation abnormalities and altered vascular integrity are also seen. 


\section{Conclusion}

Thrombocytopenia is root cause of abnormal bleeding in most of the cases. Over the years of clinical practice, physicians accurately diagnosed the varied causes of thrombocytopenia with the help of pathologists. Importance of early and prompt diagnosis of thrombocytopenia is always warranted as treatment differs depending upon the etiology. Thrombocytopenia is multifactorial and a finding that may result from various diseases. Hence the clinicopathological approach is important in thrombocytopenia cases.

\section{Conflict of Interest: None}

\section{References}

1. Lee GR, Foerster J, Lukens J. Wintrobe's Clinical Hematology, 10th ed (2 vol); Lippincott Williams \& Wilkins, 1999.

2. Rutherford CJ, Frenkel EP, Common bleeding and clotting disorders. The Med Clin N Am 1994;78(3):55575.

3. Agarwal MB: Heamatology today. Souvenir of $\mathrm{X}^{\text {th }}$ National CME in Hematology 2004.

4. Firkin F, Chesterman C, Penington D, Rush B: de Gruchy's Clinical haematology in medical practice, 5th ed; Blackwell science, 2002.

5. Lakhotia M, Shah PKD, Balani V et al: Incidence of megaloblastic anemia in Indian adults and the role of serum LDH as a diagnostic tool. Indian J Haematol Blood Transf 1994;12:113-15.

6. Harker LA: Platelets: In: Hoffbrand, Brain, Hirsh: Recent advances in hematology; Churchill Livengstone, 1977.

7. Bithell: Platelets and megakaryocytes: In: Leavell and Thorup's Fundamentals of clinical hematology; 5th ed; edited by Thorup OA et al; W.B. Saunders company; 1987.

8. Mckenzie SB: Textbook of hematology; Williams \& Wilkins; $2^{\text {nd }}$ ed; 1996.

9. Stephan F, Hollande J, Richard O et al: Thrombocytopenia in surgical ICU. Chest 1999;115(5):1363-70.
10. Ross, Manohar S, Ray S et al: A Clinical study of thrombocytopenia. JAPI 1991;39(1): 59.

11. Alam M, Mubarik A, Ahmed M et al: Clinical spectrum of thrombocytopenia in adult population of Karachi. Abstract in J coll Physians surg Pak 2001;11(10):603.

12. Pitney WR: The purpura: In: Hardisty RM, Weatherall DJ: Blood \& its disorders; Blackwell; 1974:995-1020.

13. Guida JD, Kunig AM, Leef KH et al: Platelet count and sepsis in very low birth weight neonates: is there an organism-specific response. Pediatr 2003;111(61);141115 .

14. Burstein SA: Thrombocytopenia due to decreased platelet production: In : Hoffman, Benz, Shattil et al. Heamatology: Basic principles \& practice: Churchill livingstone; $3^{\text {rd }}$ ed, 2000;2115-25.

15. George JN, Aster RH: Thrombocytopenia due to diminished or defective platelet production: In: William WJ, Beutler E, Erslev A, Lichtman MA: Hematology; MCGraw-Hill publishing company $4^{\text {th }}$ ed; $1991 ; 1343-50$.

16. Bijlani RL: Understanding of medical physiology; Jaypee brothers, 1995, 100.

17. Jandl JH: Blood: Textbook of hematology. Little, Brown and company; 1st ed; 1987.

18. Van Vilet HHD, Kappers- Klunne MC, Ables J: Pseudothrombocytopenia: a cold antibody against platelet glycoprotein GPIIb. Br J Haematol 1986,62:501.

19. Tocantis LM: The blood platelets- past, present and future: In: Johnson SA, Monto RW, Rebuck JW, Horn RC: Blood platelets: Henry Ford hospital international symposium, 1st ed; Little, Brown and company, Boston; 1961.

20. Heilmann E, Friese P, Anderson S et al: Biotinylated platelets: a new approach to the measurement of platelet life span. Br J Haematol 1993;85:729-35.

How to cite the article: Baheti M, Nikumbh D, Desai S. Clinicopathological overview of thrombocytopenia: A retrospective study in tertiary care hospital. Arch Cytol Histopathol Res 2018;3(4):214-218. 